\title{
CTBP1 strengthens the cisplatin resistance of gastric cancer cells by upregulating RAD51 expression
}

\author{
YULUO WU $^{1}$ and HAIYANG ZHAO ${ }^{2}$ \\ ${ }^{1}$ Department of Oncology, Guangdong Medical University Affiliated Central People's Hospital of Zhanjiang, Zhanjiang, \\ Guangdong 524000; ${ }^{2}$ Department of Oncology, Beijing Zhongguancun Hospital, Beijing 100190, P.R. China
}

Received April 17, 2021; Accepted July 16, 2021

DOI: 10.3892/ol.2021.13071

\begin{abstract}
Drug resistance is a key factor affecting the treatment of gastric cancer. The resistance of gastric cancer cells to anticancer drugs, such as cisplatin (DDP), remains a major challenge to patient recovery. The present study aimed to investigate the roles of C-terminal-binding protein 1 (CTBP1) in the DDP resistance of gastric cancer cells and to determine its regulatory effect on DNA repair protein RAD51 homolog 1 (RAD51). The DDP-resistant human gastric cancer AGS and HGC cell lines, AGS/DDP and HGC-27/DDP, respectively, were established and CTBP1 expression was detected by western blotting. In addition, Cell Counting Kit-8, colony formation and flow cytometry assays were performed to detect the proliferation and apoptosis of these two cell lines following CTBP1 knockdown. The expression levels of apoptosis-related proteins were detected by western blotting. In addition, RAD51 was overexpressed in CTBP1 knockdown cells, and proliferation and apoptosis were subsequently determined using the aforementioned methods. The results demonstrated that CTBP1 expression was notably increased in DDP-resistant gastric cancer cells. Furthermore, CTBP1 knockdown suppressed the proliferation and induced the apoptosis of AGS/DDP and HGC-27/DDP cells. Notably, CTBP1 promoted RAD51 expression in DDP-resistant gastric cancer cells. Overexpression of RAD51 in CTBP1 knockdown AGS/DDP and HGC-27/DDP cells rescued the proliferation and alleviated the apoptosis of these cells. Taken together, the results of the present study suggested that CTBP1 may enhance the DDP resistance of gastric cancer cells by activating RAD51 expression, thus providing a potential novel therapy (CTBP1 knockdown) for the clinical treatment of patients with gastric cancer.
\end{abstract}

Correspondence to: Dr Haiyang Zhao, Department of Oncology, Beijing Zhongguancun Hospital, 12 Zhongguancun South Road, Haidian, Beijing 100190, P.R. China

E-mail: zhaohaiyzhy@163.com

Key words: gastric cancer, cisplatin resistance, C-terminal-binding protein 1, DNA repair protein RAD51 homolog 1, proliferation

\section{Introduction}

Gastric cancer originates from the gastric mucosa and is one of the most common malignancies worldwide (1). Gastric cancer is associated with high mortality rates, and as per 2018 GLOBOCAN data, gastric cancer constitutes $8.2 \%$ of all cancer-related mortalities, which continues to increase the burden on the global public health system (2). The main risk factors of gastric cancer include a poor diet, a sedentary lifestyle and Helicobacter pylori infection (3). Despite recent advancements in treatment strategies, the 5-year survival rate of patients with gastric cancer remains $<10 \%$, and metastasis of gastric cancer cells is the main cause of mortality $(4,5)$.

Cisplatin (DDP) is a common anticancer drug used to treat gastric cancer (6). Most gastric cancer cells are highly resistant to DDP and the further development of drug resistance limits the cytotoxic effect of DDP on gastric cancer cells (7). Thus, it is important to investigate the molecular mechanisms underlying drug resistance in gastric cancer cells to provide novel strategies for the clinical treatment of this disease. C-terminal-binding protein 1 (CTBP1) is expressed in different types of cancer, such as lung adenocarcinoma, endometrial carcinoma and glioma (8-10). Previous studies have reported that CTBP1 is the transcriptional corepressor of several tumor suppressor genes $(11,12)$, and various physiological processes, such as proliferation, apoptosis and epithelial-to-mesenchymal transition (EMT), in different types of cells, such as cancer cells and cancer stem cells, are regulated by CTBP1 (13). CTBP1 expression affects cell proliferation, migration and invasion of different types of cancer cells. For example, CTBP1 knockdown suppresses the proliferation and invasion of breast cancer cells by repressing the EMT process (14). Furthermore, during the development of gastric cancer, microRNA (miR)-539-3p inhibits the migration and invasion of gastric tumor cells by suppressing CTBP1 expression (15). Notably, high levels of CTBP1 can also promote the proliferation, migration and invasion of gastric tumor cells (16). Importantly, CTBP1 is reported to confer DDP resistance to breast cancer cells (17). However, whether CTBP1 could regulate the DDP resistance of gastric cancer remains unknown.

DNA repair protein RAD51 homolog 1 (RAD51) is a protein associated with DNA damage repair (18). However, RAD51 expression is also associated with the development of different types of cancer, such as ovarian, colorectal and 
breast cancer (19-21). STAT5A/B inhibition increases the sensitivity of prostate cancer cells to radiotherapy through inhibition of RAD51 and DNA repair (22). In addition, CTBP1 can enhance the resistance of breast cancer cells to DDP by activating RAD51 expression (17). However, whether RAD51 expression affects the resistance of gastric cancer cells to DDP remains unclear. Thus, the present study aimed to investigate the effects of CTBP1 and RAD51 on the DDP resistance of gastric cancer cells.

\section{Materials and methods}

Cell culture and treatment. Human gastric cancer cell lines (AGS and HGC-27), the corresponding DDP-resistant AGS and HGC-27 cell lines (AGS/DDP and HGC-27/DDP) and the gastric mucosal epithelial cell line (GES-1) were purchased from The Cell Bank of Type Culture Collection of The Chinese Academy of Sciences. Cells were maintained in Dulbecco's modified Eagle's medium (Thermo Fisher Scientific, Inc.) supplemented with 10\% FBS (Gibco; Thermo Fisher Scientific, Inc.). AGS/DDP and HGC-27/DDP cells were maintained in the same culture medium supplemented with various concentrations of DDP $(2,4,6,8$ and $10 \mu \mathrm{g} / \mathrm{ml}$; Thermo Fisher Scientific, Inc.). All cells were cultured at $37^{\circ} \mathrm{C}$ in a humidified atmosphere with $5 \% \mathrm{CO}_{2}$. The usage of these cells was in accordance with the previous study (23).

Cell transfection. A total of two CTBP1 small interfering (si)RNAs (siCTBP1-1 and siCTBP1-2; $50 \mathrm{nM}$ ) and the negative control (siNC; $50 \mathrm{nM}$ ), CTBP1 overexpressing pcDNA3.1 plasmid (pc-CTBP1; $50 \mathrm{nM}$ ), RAD51 overexpressing pcDNA3.1 plasmid (pc-RAD51; $50 \mathrm{nM}$ ) and empty control plasmid (pcDNA3.1; $50 \mathrm{nM}$ ) were purchased from Shanghai GeneChem Co., Ltd. Cells were plated into 6-well plates ( $1 \times 10^{6}$ cells per well), and transfection was performed when cells in the logarithmic growth phase reached $80 \%$ confluence. Transfection was performed using Lipofectamine ${ }^{\circledR} 2000$ reagent (Invitrogen; Thermo Fisher Scientific, Inc.) at $37^{\circ} \mathrm{C}$ for $48 \mathrm{~h}$, according to the manufacturer's instructions. Transfection efficiency was assessed by western blotting after $48 \mathrm{~h}$.

Cell viability assay. The Cell Counting Kit-8 (CCK-8; Shanghai Yi Sheng Biotechnology Co., Ltd.) assay was performed to detect cell viability. Cell suspensions $\left(2 \times 10^{3}\right.$ cells) were plated into 96-well plates. Following adhesion of cells (90\% confluency), CCK- 8 reagent was diluted with the culture medium and added into each well for $4 \mathrm{~h}$. The absorbance at $450 \mathrm{~nm}$ was measured using a microplate reader (Molecular Devices, LLC).

Colony formation assay. Cell suspensions were plated into $60-\mathrm{mm}$ culture dishes at a density of 300 cells/dish. Cells were cultured in the incubator for 2 weeks, and subsequently fixed with $70 \%$ ethanol for $40 \mathrm{~min}$ at room temperature and stained with crystal violet (Thermo Fisher Scientific, Inc.) for $5 \mathrm{~min}$ at room temperature. Groups of $>50$ cells were considered a clone and were assessed using Image J software (version 1.52r; National Institutes of Health). Stained cells were observed under a light microscope (Olympus Corporation).
Apoptosis analysis. Apoptosis was detected by flow cytometric analysis. Cell suspensions $\left(1 \times 10^{6}\right)$ were washed three times with PBS to clear residual FBS from the culture medium. Cells were subsequently incubated with Annexin V-FITC and propidium iodide (PI) using an Annexin V-FACS apoptosis detection kit (Beyotime Institute of Biotechnology) at $37^{\circ} \mathrm{C}$ in humidified atmosphere for $1 \mathrm{~h}$. Cells were washed three times with PBS, and apoptotic cells were subsequently analyzed using a FACSAria flow cytometer (Thermo Fisher Scientific, Inc.) with FlowJo software (version 10.0; FlowJo LLC; BD Bioscience).

Reverse transcription-quantitative PCR (RT-qPCR). Total RNA was extracted from $1 \times 10^{6}$ cells using TRIzol ${ }^{\circledR}$ reagent (Thermo Fisher Scientific, Inc.), and reverse transcribed into cDNA using a PrimeScript ${ }^{\mathrm{TM}}$ RT Reagent kit (Takara Bio, Inc.) according to the manufacturer's protocol. cDNA was subsequently amplified using an SYBR Green RT-PCR kit on an Applied Biosystems 7500 Real-Time PCR system (Thermo Fisher Scientific, Inc.) and relative expression levels were calculated using the $2^{-\Delta \Delta \mathrm{Cq}}$ method (24). The following thermocycling conditions were used for qPCR: Initial denaturation for $10 \mathrm{~min}$ at $95^{\circ} \mathrm{C}$, followed by 40 cycles of denaturation at $95^{\circ} \mathrm{C}$ for $15 \mathrm{sec}$ and annealing/extension at $55^{\circ} \mathrm{C}$ for $45 \mathrm{sec}$. GAPDH expression level was used as a reference control. The following primer sequences were used for qPCR: CTBP1 forward, 5'-CGACCCTTACTTGTCGGATGG-3' and reverse, 5'-TTGACGGTGAAGTCGTTGATG-3'; RAD51 forward, 5'-CTATGTAGCAAAGGGAATGGG-3' and reverse, 5'-AAG CAGGTAGATGGTGAAGG-3'; and GAPDH forward, 5'-GGAGCGAGATCCCTCCAAAAT-3' and reverse, 5'-GGC TGTTGTCATACTTCTCATGG-3'.

Western blotting. Total protein was extracted from $1 \times 10^{6}$ cells using RIPA lysis buffer (Beyotime Institute of Biotechnology). Total protein was quantified using a bicinchoninic acid assay (Beyotime Institute of Biotechnology) and proteins (40 $\mu \mathrm{g}$ protein/lane) were separated on $10 \%$ gels using SDS-PAGE. The separated proteins were subsequently transferred onto PVDF membranes and blocked with $5 \%$ skimmed milk powder in Tris-buffered saline overnight at $4^{\circ} \mathrm{C}$. The membranes were incubated with primary antibodies against CTBP1 (cat. no. ab129181; 1:1,000; Abcam), RAD51 (cat. no. ab133534; 1:1,000; Abcam), Bax (cat.no. ab32503; 1:1,000; Abcam), Bcl-2 (cat. no. ab182858; 1:1,000; Abcam), cleaved (c)-caspase-3 (cat. no. 9664T; 1:1,000; Cell Signaling Technology, Inc.), caspase-3 (cat. no. 14220T; 1:1,000; Cell Signaling Technology, Inc.), c-caspase-9 (cat. no. 20750S; 1:1,000; Cell Signaling Technology, Inc.), caspase-9 (cat. no. 9508T; 1:1,000; Cell Signaling Technology, Inc.) and GAPDH (cat. no. ab8245; 1:1,000; Abcam) overnight at $4^{\circ} \mathrm{C}$. Following the primary incubation, membranes were incubated with horseradish peroxidase-conjugated goat anti-rabbit secondary antibody (cat. no. ab205718; 1:2,000; Abcam) or anti-mouse horseradish peroxidase-conjugated secondary antibody (cat. no. ab205719; 1:5,000; Abcam) at room temperature for $2 \mathrm{~h}$. Protein bands were visualized using Pierce ECL Western Blotting Substrate (Thermo Fisher Scientific, Inc.). GAPDH was used as the reference protein. Protein expression was quantified using ImageJ software (version 1.41; National Institutes of Health). 
A
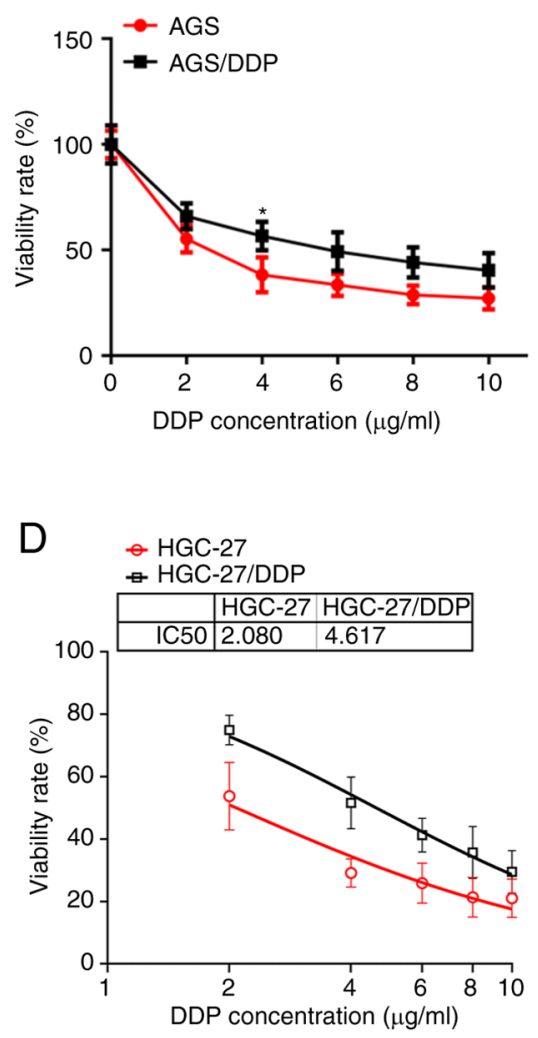

B

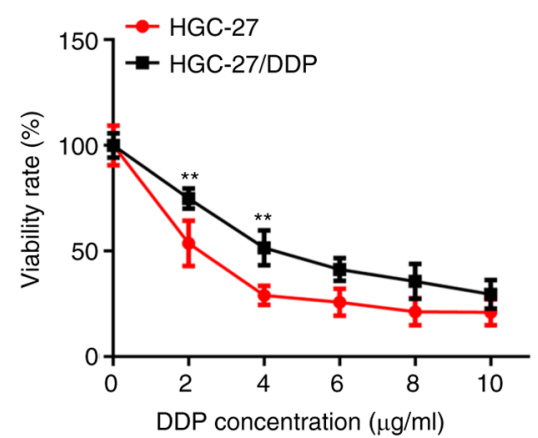

E

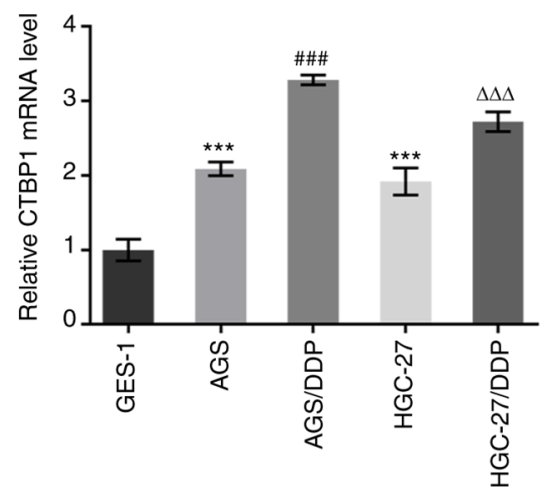

C

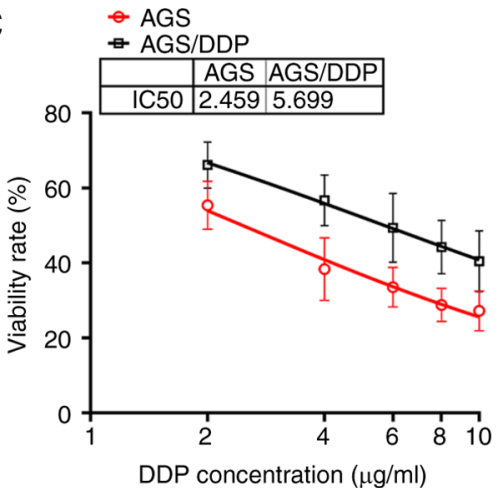

$\mathrm{F}$
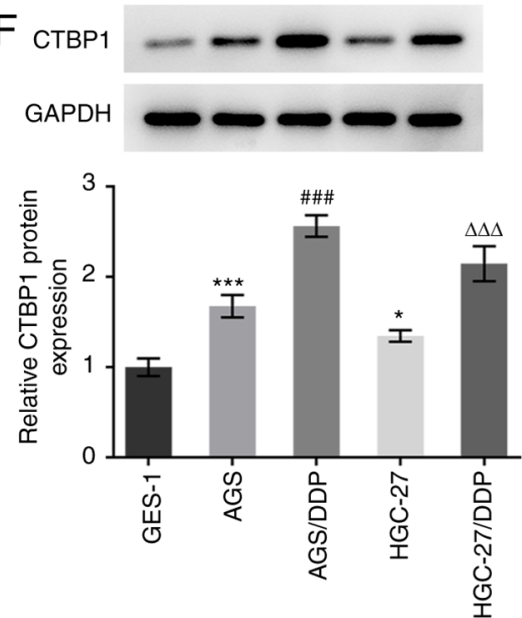

Figure 1. CTBP1 expression is upregulated in DDP-resistant gastric cancer cells. Viability of DDP-resistant gastric cancer cells. (A) AGS/DDP and (B) HGC-27/DDP, using the Cell Counting Kit-8 assay. ${ }^{*} \mathrm{P}<0.05,{ }^{*} \mathrm{P}<0.01$ vs. AGS or HGC-27. The $\mathrm{IC}_{50}$ values of DDP in (C) AGS/DDP and (D) HGC-27/DDP cells were detected by CCK-8 assay. (E) Reverse transcription-quantitative PCR analysis was performed to detect CTBP1 mRNA expression and (F) western blot analysis was performed to detect CTBP1 protein expression in gastric cancer and normal gastric mucosal epithelial cells. ${ }^{*} \mathrm{P}<0.05,{ }^{* * *} \mathrm{P}<0.001$ vs. GES-1;

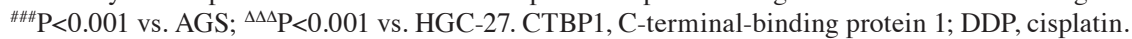

Statistical analysis. Statistical analysis was performed using GraphPad Prism 8.0 software (GraphPad Software, Inc.). All experiments were performed in triplicate and data are presented as the mean \pm SD. One-way ANOVA followed by Tukey's post hoc test was used to compare differences between multiple groups. Pearson's correlation coefficient was used to assess the correlation between CTBP1 and RAD51 mRNA expression. $\mathrm{P}<0.05$ was considered to indicate a statistically significant difference.

\section{Results}

CTBP1 expression is upregulated in DDP-resistant gastric cancer cells. Drug resistance of gastric cancer cells was verified using the CCK-8 viability assay. As demonstrated in Fig. $1 \mathrm{~A}$ and $\mathrm{B}$, the viability of DDP-resistant gastric cancer cells, AGS/DDP ( $4 \mu \mathrm{g} / \mathrm{ml})$ and HGC-27/DDP (2 and $4 \mu \mathrm{g} / \mathrm{ml})$, was higher compared with that of gastric cancer cells, AGS and HGC-27. The $\mathrm{IC}_{50}$ values of DDP in AGS/DDP and HGC-27/DDP cells were determined, which showed that the $\mathrm{IC}_{50}$ of DDP was higher in AGS/DDP and HGC-27/DDP cells compared with that in AGS and HGC-27 cells (Fig. 1C and D). Subsequently, CTBP1 expression was detected in gastric cancer cells and normal gastric mucosal epithelial cells.
CTBP1 mRNA and protein expression levels were significantly higher in AGS and HGC-27 cells compared with GES-1 cells (Fig. 1E and F). Notably, CTBP1 expression was higher in AGS/DDP and HGC-27/DDP cells compared with expression in AGS and HGC-27 cells. Taken together, these results suggested that CTBP1 expression is significantly upregulated in DDP-resistant gastric cancer cells.

CTBP1 knockdown inhibits the proliferation of DDP-resistant gastric cancer cells. To detect the effect of CTBP1 on the proliferation of DDP-resistant gastric cancer cells, CTBP1 expression was silenced by transfecting cells with siCTBP1-1 or siCTBP1-2. As presented in Fig. 2A and D, CTBP1 protein expression levels decreased in AGS/DDP and HGC-27/DDP cells following transfection. Transfection with siCTBP1-2 decreased CTBP1 expression to the greatest extent; therefore, siCTBP1-2 was selected for subsequent experimentation. Results from the CCK- 8 assay demonstrated that CTBP1 knockdown significantly decreased the viability of AGS/DDP and HGC-27/DPP cells compared with that of the respective siNC-transfected group (Fig. 2B and E). The inhibitory effects of CTBP1 knockdown on AGS and HGC-27 cell viability are presented in Fig. S1. Similarly, the results of the colony formation assay demonstrated that the number of cell colonies in 

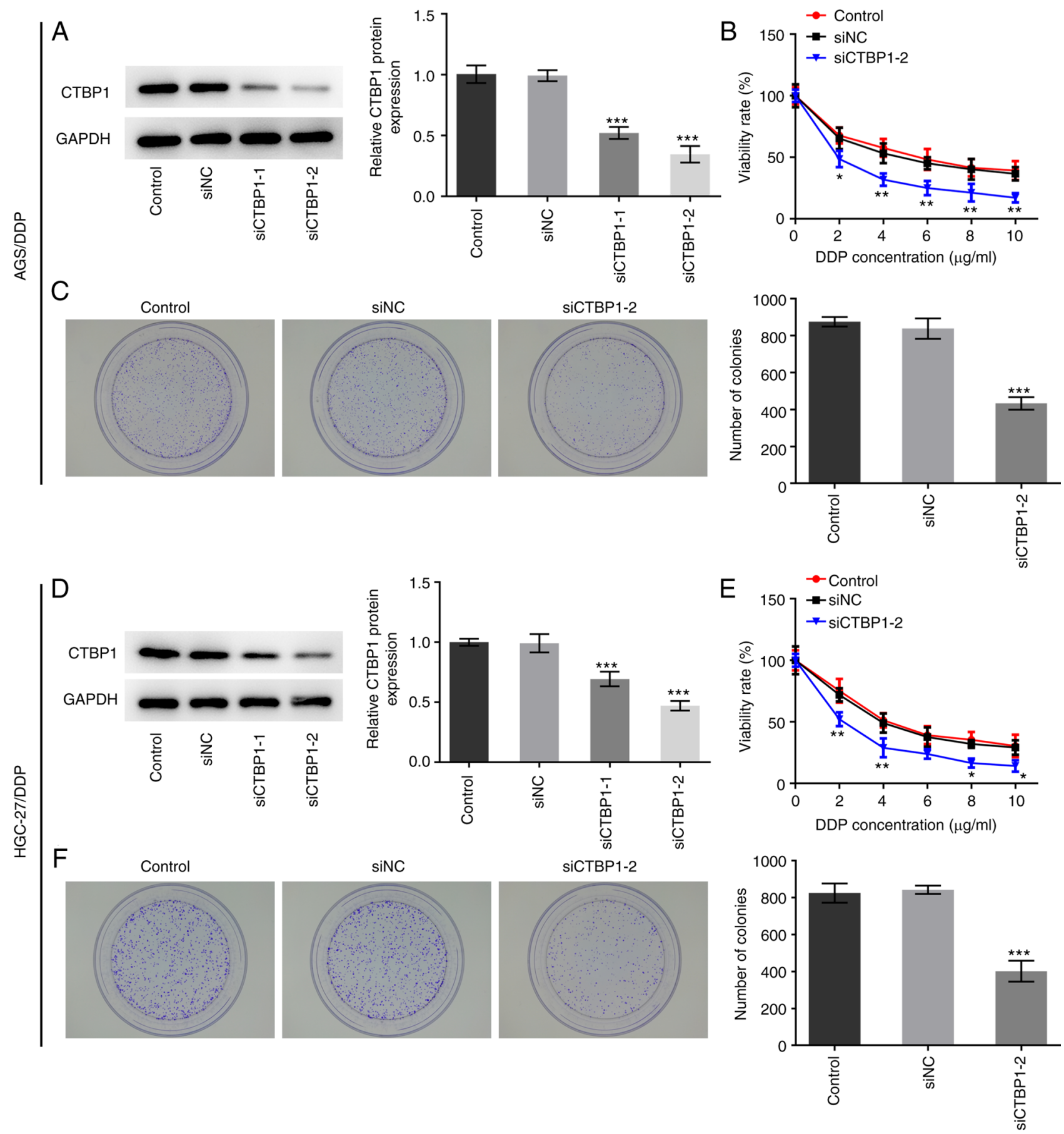

Figure 2. CTBP1 knockdown suppresses the proliferation of DDP-resistant gastric cancer cells. (A) Western blot analysis was performed to detect CTBP1 protein expression in AGS/DDP cells. (B) The CCK-8 assay was performed to detect the viability of AGS/DDP cells. (C) The colony formation assay was performed to detect the proliferation of AGS/DDP cells. (D) Western blot analysis was performed to detect CTBP1 protein expression in HGC-27/DDP cells. The (E) CCK-8 and (F) colony formation assays were performed to detect the proliferation of HGC-27/DDP cells. " $\mathrm{P}<0.05$, ${ }^{* * *} \mathrm{P}<0.01,{ }^{* * * *} \mathrm{P}<0.001$ vs. siNC. CCK-8, Cell Counting Kit-8; CTBP1, C-terminal-binding protein 1; DDP, cisplatin; NC, negative control; si, small interfering RNA.

DDP-resistant AGS and HGC-27 cells significantly decreased following CTBP1 knockdown (Fig. 2C and F). Collectively, these results suggested that CTBP1 knockdown suppressed the proliferation of cisplatin-resistant gastric cancer cells.

CTBP1 knockdown promotes the apoptosis of DDP-resistant gastric cancer cells. The effect of CTBP1 on the apoptosis of DDP-resistant gastric cancer cells was examined. The apoptotic rates of AGS/DDP and HGC-27/DDP cells were significantly increased following CTBP1 knockdown compared with the respective siNC group (Fig. 3A and $\mathrm{C}$, respectively). The expression levels of the apoptosis-related proteins were detected by western blotting. As presented in Fig. 3B and D, CTBP1 knockdown in AGS/DDP and HGC-27/DDP cells significantly decreased Bcl-2 expression but increased Bax, c-caspase-3 and c-caspase-9 expression compared with the respective siNC group. Taken together, these results suggested that CTBP1 knockdown accelerated the apoptosis of DDP-resistant gastric cancer cells. 


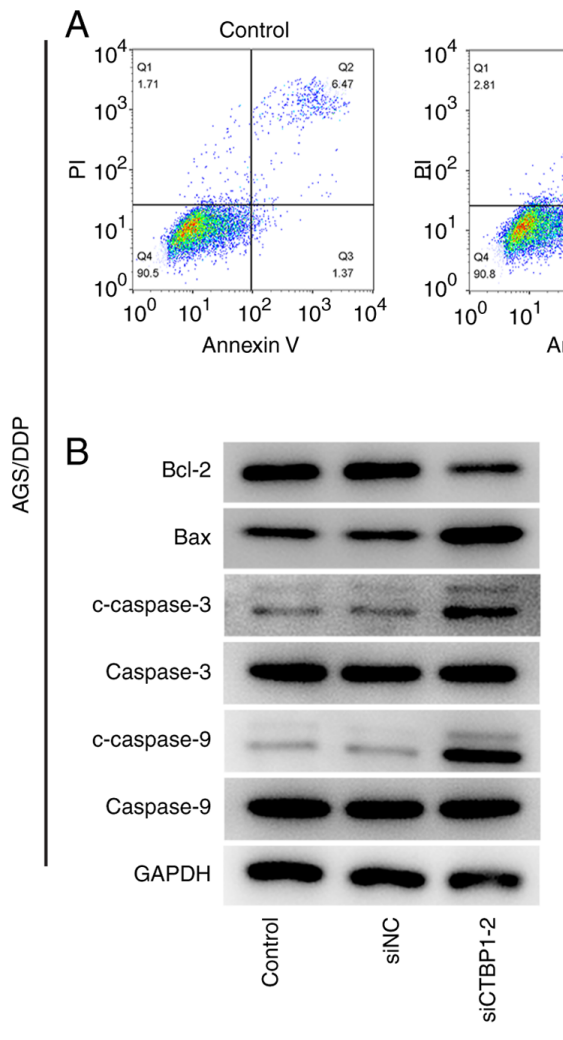

siNC

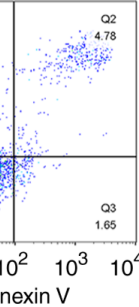

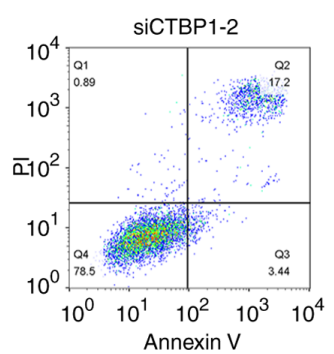
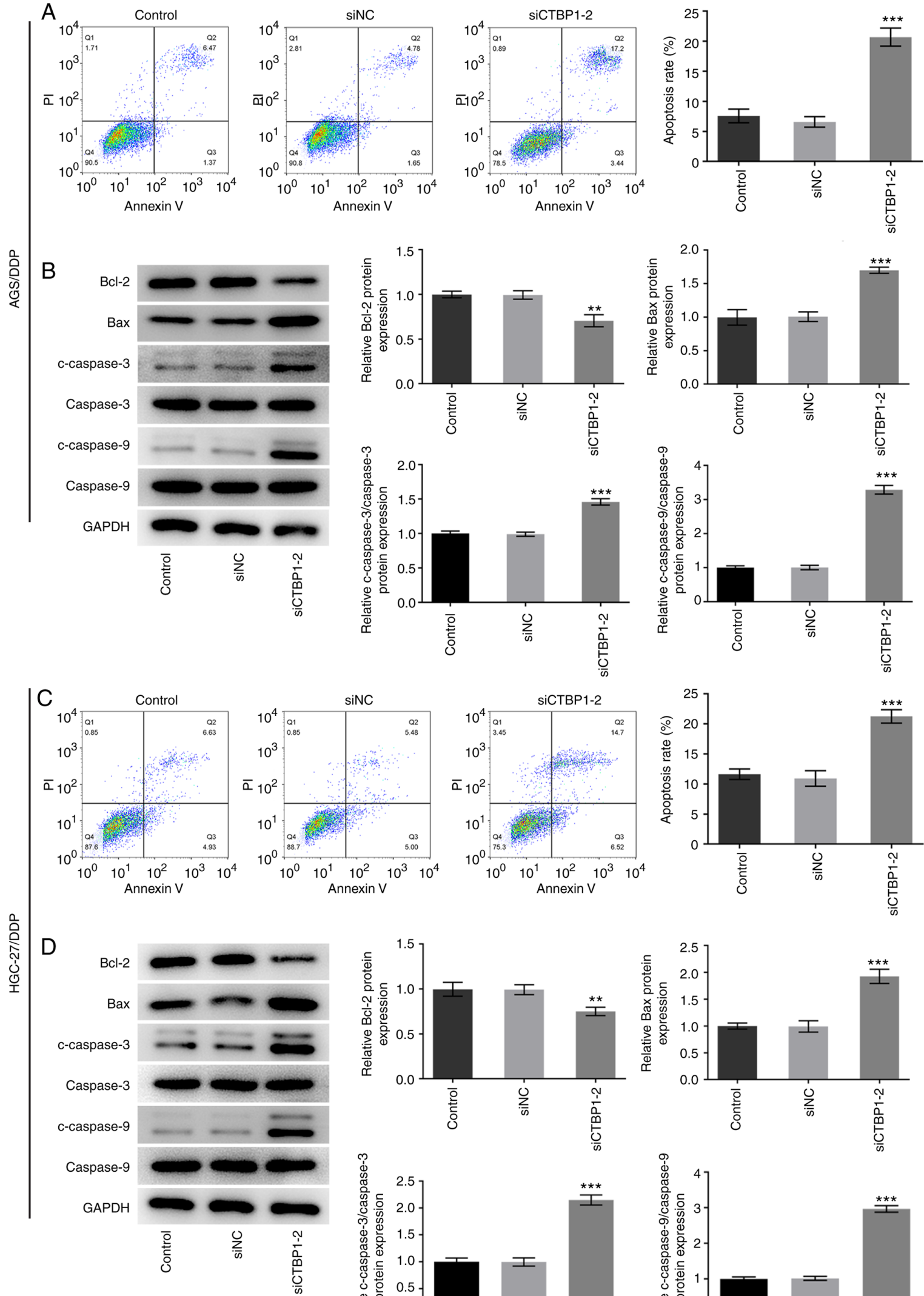

siNC
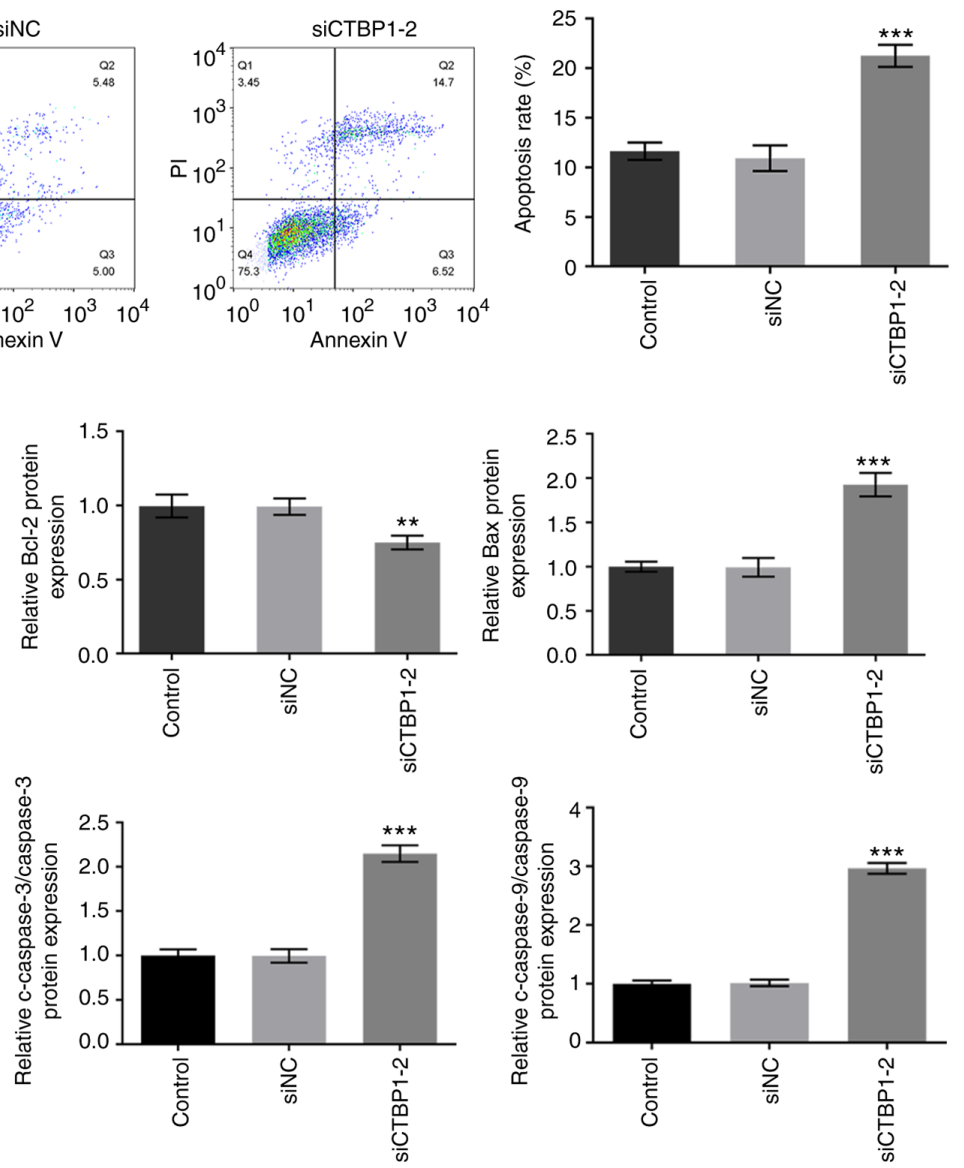

Figure 3. CTBP1 knockdown induces the apoptosis of DDP-resistant gastric cancer cells. (A) Flow cytometric analysis was performed to detect the apoptosis of AGS/DDP cells. (B) Western blot analysis was performed to detect the protein expression levels of apoptosis-related proteins in AGS/DDP cells. (C) Flow cytometric analysis was performed to detect the apoptosis of HGC-27/DDP cells. (D) Western blot analysis was performed to detect the protein expression levels of apoptosis-related proteins in HGC-27/DDP cells. ${ }^{* *} \mathrm{P}<0.01,{ }^{* * *} \mathrm{P}<0.001$ vs. siNC. CTBP1, C-terminal-binding protein 1 ; DDP, cisplatin; NC, negative control; si, small interfering RNA. 
A
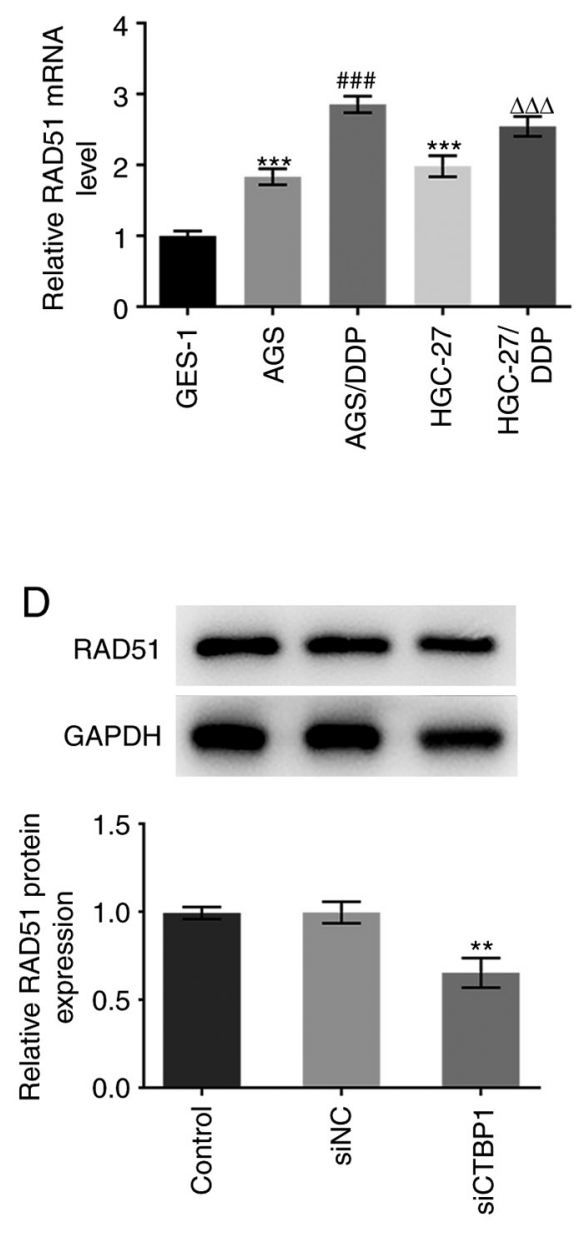

$\mathrm{B}$

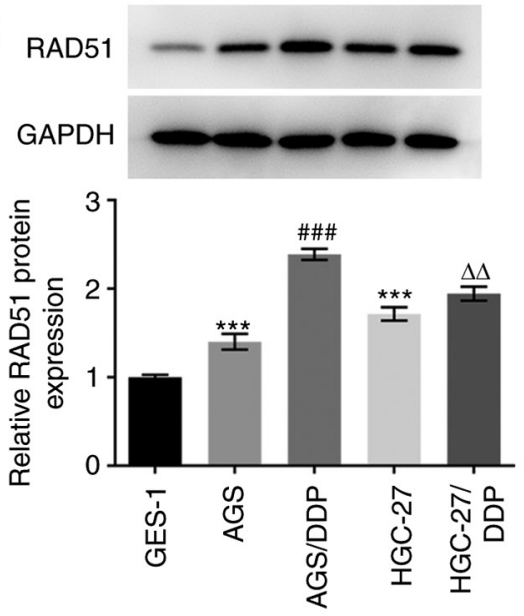

$\mathrm{E}$
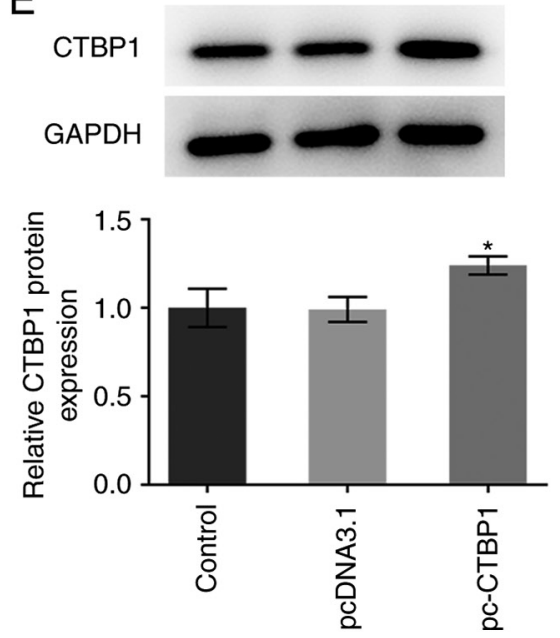

C

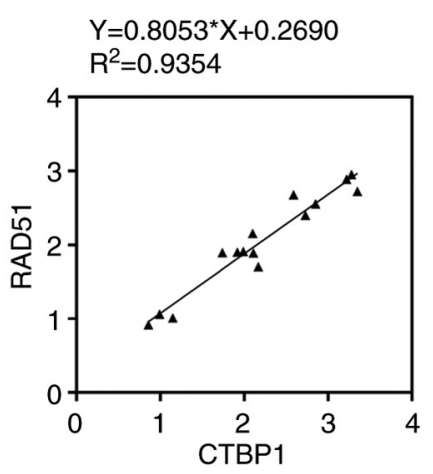

$\mathrm{F}$
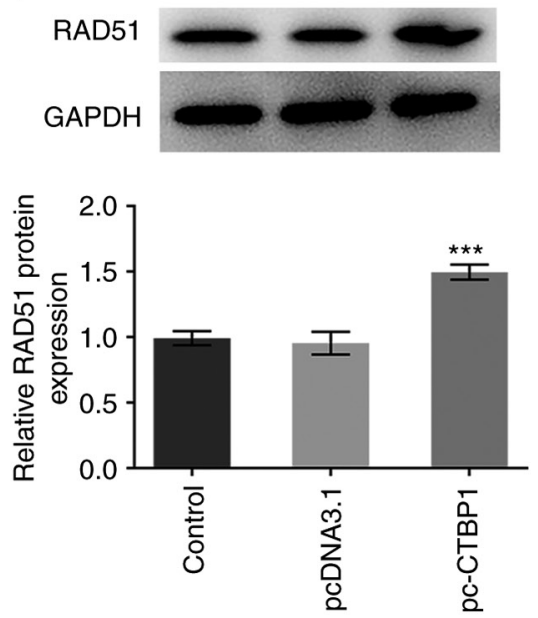

Figure 4. CTBP1 upregulated RAD51 expression in DDP-resistant gastric cancer cells. (A) RT-qPCR and (B) western blot analysis were used to determine the expression levels of RAD51 mRNA and protein, respectively. ${ }^{* * *} \mathrm{P}<0.001$ vs. GES-1; ${ }^{\# \#} \mathrm{P}<0.001$ vs. AGS; ${ }^{\Delta \Delta} \mathrm{P}<0.01,{ }^{\Delta \Delta \Delta} \mathrm{P}<0.001$ vs. HGC-27. (C) Pearson's correlation analysis between CTBP1 and RAD51 mRNA levels in GES-1, AGS, AGS/DDP, HGC-27 and HGC-27/DDP cells. (D) Western blot analysis was performed to detect RAD51 protein expression in AGS/DDP cells following CTBP1 knockdown. ${ }^{* *} \mathrm{P}<0.01 \mathrm{vs}$. siNC. (E) Western blot analysis was performed to detect CTBP1 protein expression in AGS/DDP cells following transfection with pc-CTBP1. (F) Western blot analysis was performed to detect RAD51 protein expression in AGS/DDP cells following transfection with CTBP1 plasmid. ${ }^{*} \mathrm{P}<0.05,{ }^{* * *} \mathrm{P}<0.001$ vs. pcDNA3.1. CTBP1, C-terminal-binding protein 1; DDP, cisplatin; NC, negative control; pc, pcDNA3.1 overexpression vector; RAD51, DNA repair protein RAD51 homolog 1; si, small interfering RNA.

RAD51 overexpression abolishes the anticancer effect of CTBP1 knockdown on DDP-resistant gastric cancer cells. A previous study reported that CTBP1 expression influences DDP resistance of breast cancer cells by activating RAD51 expression (19). In the present study, RT-qPCR and western blot analysis were used to evaluate the expression of RAD51 in the aforementioned five cell lines. RAD51 mRNA and protein expression levels were significantly upregulated in the AGS and HGC-27 cells compared with expression levels in the GES-1 cells (Fig. 4A and B); in addition, RAD51 expression was higher in the AGS/DDP and HGC-27/DDP cells compared with that in the AGS and HGC-27 cells. Subsequently, the correlation between CTBP1 and RAD51 mRNA expression levels was assessed, and a positive correlation was revealed between CTBP1 and RAD51 mRNA expression (Fig. 4C). AGS/DPP cells exhibited higher CTBP1 and RAD51 expression levels; therefore, this cell line was selected to perform the subsequent experiments. RAD51 expression was notably decreased in AGS/DDP cells following CTBP1 knockdown (Fig. 4D). Following overexpression of CTBP1, western blot analysis demonstrated that CTBP1 expression was markedly increased in the overexpression group (Fig. 4E). As presented in Fig. 4F, elevated CTBP1 expression significantly increased RAD51 expression.

To further determine the association between CTBP1 and RAD51 in gastric cancer, RAD51 overexpressing plasmid was transfected into AGS/DDP cells (Fig. 5A). A notably reduced CTBP1 protein expression level was observed in the siCTBP1-2 group compared with the siNC group, and there was no significant difference in CTBP1 expression between the siCTBP1-2 + pcDNA3.1 and the siCTBP1-2 + pc-RAD51 groups (Fig. 5B). However, cells transfected with siCTBP1-2 exhibited a significant downregulation in RAD51 protein expression in comparison to the siNC group, which was partially restored by co-transfection with pc-RAD51 (Fig. 5B). In addition, the results demonstrated that overexpression of RAD51 alleviated the inhibitory effects of siCTBP1 transfection on the viability and proliferation of AGS/DDP cells (Fig. 5C and D, respectively). Similarly, overexpression of RAD51 relieved CTBP1 knockdown-induced apoptosis of DDP-resistant AGS 
A

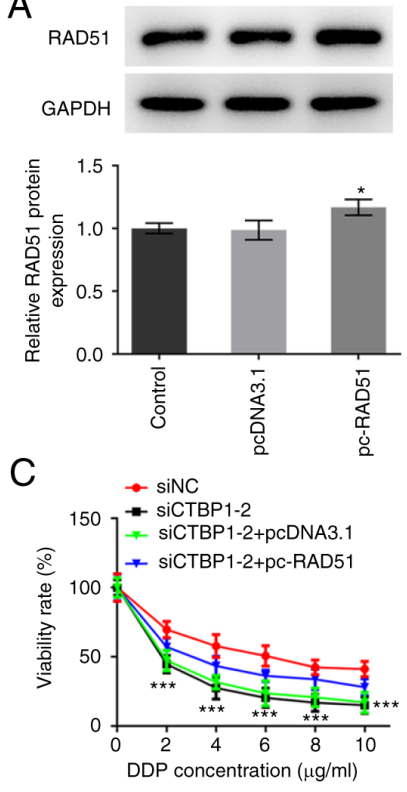

E
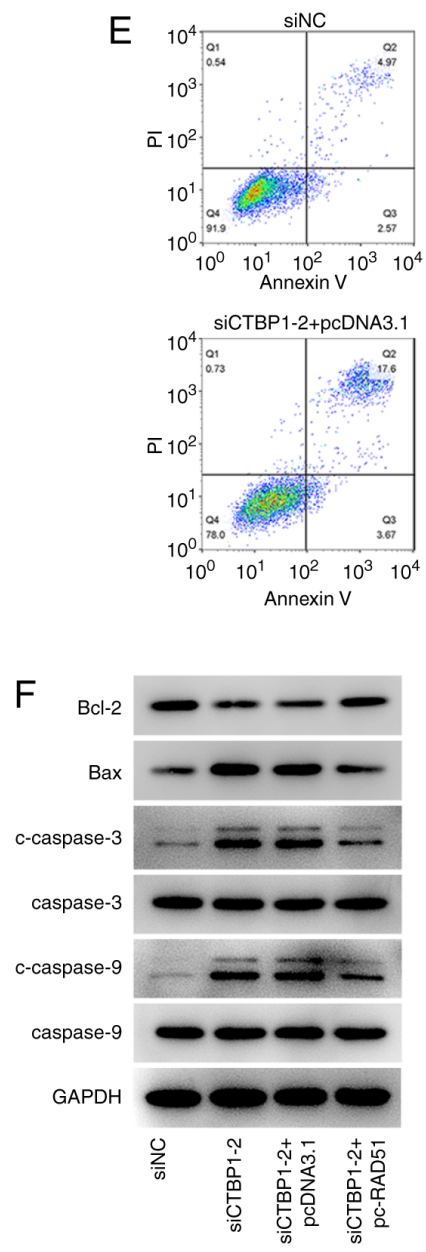

B
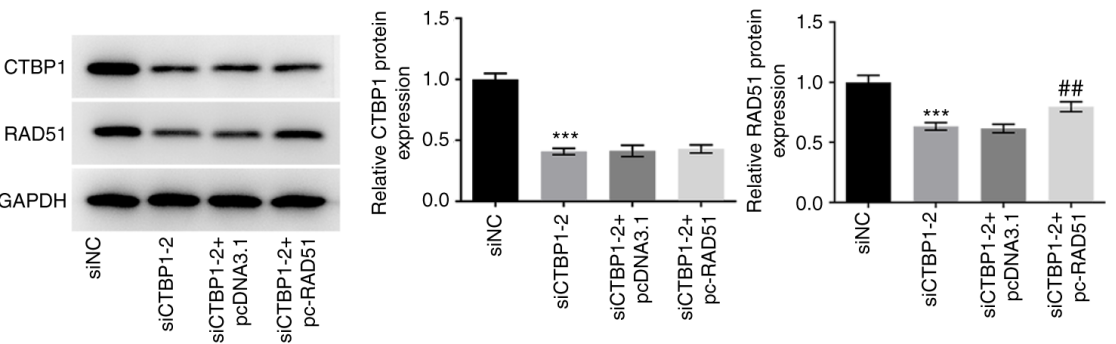

D

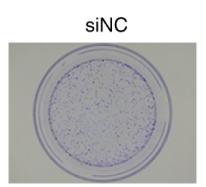

siCTBP1-2+pcDNA3.1

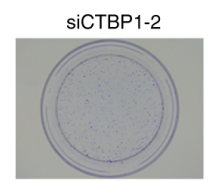

siCTBP1-2+pc-RAD51
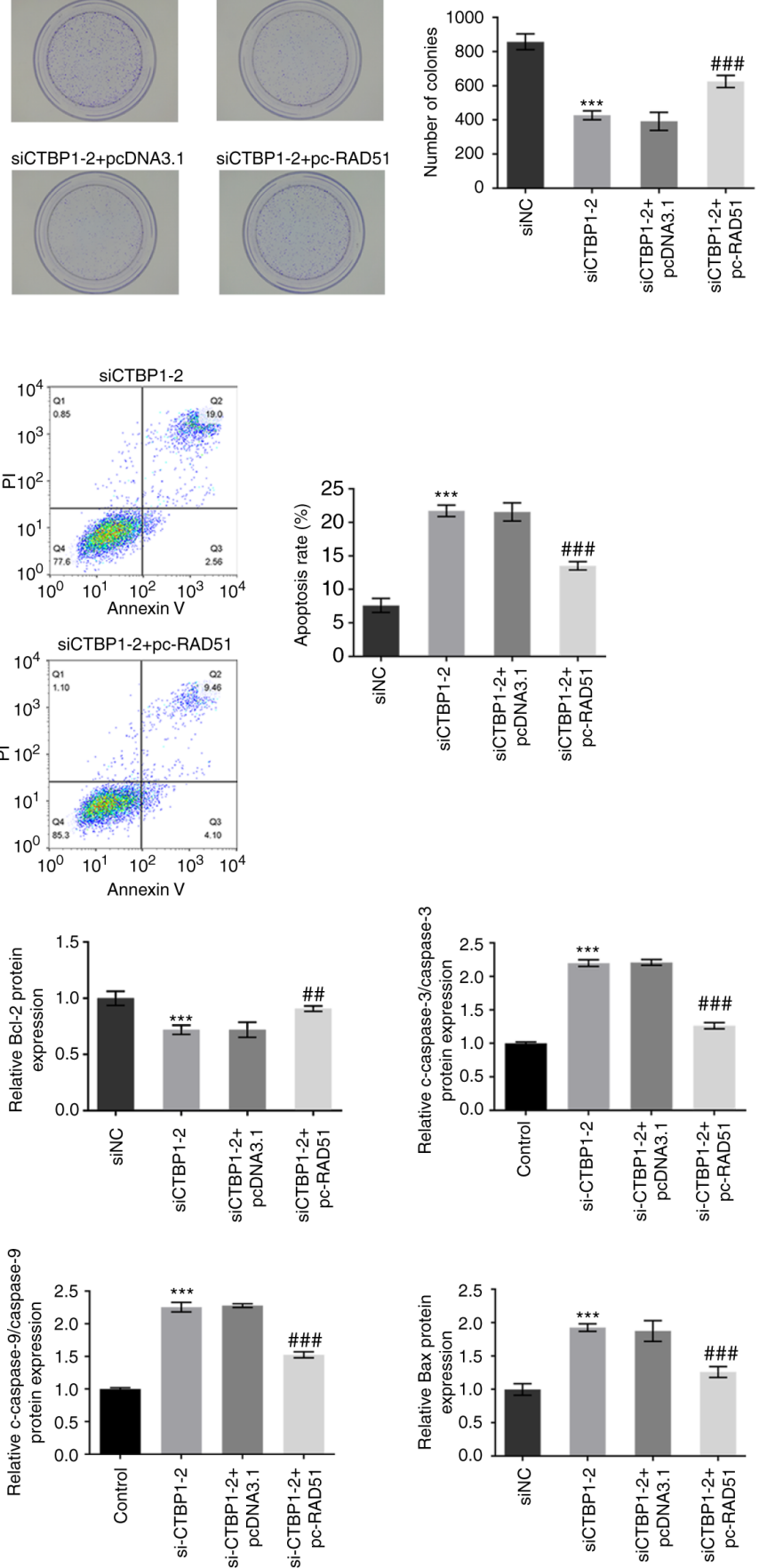

Figure 5. Overexpression of RAD51 abolishes CTBP1 knockdown-induced effects on proliferation and apoptosis of DDP-resistant gastric cancer cells. (A) Western blot analysis was performed to detect RAD51 protein expression levels in AGS/DDP cells following transfection with RAD51 plasmid. * $<0.05$ vs. pcDNA3.1. (B) The protein expression levels of CTBP1 and RAD51 following transfection with siCTBP1-2 with or without pc-RAD51 co-transfection was determined using western blot analysis. (C) Viability of AGS/DDP cells were evaluated using the Cell Counting Kit-8 assay. (D) Colony formation assay was performed to detect the proliferation of AGS/DDP cells. (E) Flow cytometric analysis was performed to detect the apoptosis of AGS/DDP cells. (F) Western blot analysis was performed to detect the protein expression levels of apoptosis-related proteins in AGS/DDP cells. ${ }^{* * *} \mathrm{P}<0.001$ vs. siNC; ${ }^{\# \#} \mathrm{P}<0.01,{ }^{\# \# \# P<0.001}$ vs. siCTBP1-2 + pcDNA3.1. CTBP1, C-terminal-binding protein 1; DDP, cisplatin; RAD51, DNA repair protein RAD51 homolog 1; si, small interfering RNA. 
cells (Fig. 5E). Notably, Bcl-2 protein expression significantly increased, whereas Bax, c-caspase-3 and c-caspase-9 expression levels decreased following overexpression of RAD51 in CTBP1 knockdown AGS/DDP cells when compared with the siCTBP1-2 + pcDNA3.1 group (Fig. 5F). Collectively, these results suggested that CTBP1 may regulate DDP resistance in gastric cancer cells by modulating RAD51 expression.

\section{Discussion}

Gastric cancer is a common malignancy of the digestive system (2). Despite advancements in treatment strategies, gastric cancer is associated with high mortality rates (25), which is predominantly due to the biological characteristics of gastric cancer, such as the delayed clinical manifestations, and the proliferation and metastasis of gastric cancer cells (26). Several anticancer drugs, such as DDP, exert strong antitumoral effects on gastric cancer cells $(27,28)$. However, the enhanced resistance of gastric cancer cells to DDP and other drugs gradually weaken the corresponding anticancer drug treatment effects (29). Thus, it is important to determine the molecular mechanisms underlying DDP resistance of gastric cancer cells.

As a transcription factor, CTBP1 serves a crucial role in regulating cell proliferation and apoptosis in different types of cells, such as breast cancer, osteosarcoma and ovarian cancer (14,30-32). Furthermore, CTBP1 enhances the occurrence and development of different types of cancer (26). A previous study reported that CTBP1 promotes the invasion and development of tumor-associated macrophages during the development of non-small cell lung cancer (27). Furthermore, overexpression of CTBP1 can promote the development of breast cancer by inducing occurrence and the EMT process (13). During the development of gastric cancer, miR-644a inhibits the proliferation and invasion of gastric cancer cells by suppressing CTBP1 expression (28). Furthermore, high levels of CTBP1 induce the migration and invasion of gastric mucosal epithelial cells (15). The results of the present study suggested that CTBP1 has the potential to enhance the development of gastric cancer; CTBP1 knockdown suppressed the proliferation and induced the apoptosis of DDP-resistant gastric cancer cells.

RAD51 is a protein observed in different types of cells, such as those of ovarian, colorectal and breast cancer, and its expression is key in the process of homologous recombination, which is one of the major mechanisms of the DNA damage response (29). When DNA damage occurs, RAD51 forms nucleofilaments with other repair proteins, such as BRCA2 and partner and localizer of BRCA2, on single-stranded DNA to facilitate homologous strand pairing and strand exchange during the DNA damage repair process $(30,31)$. RAD51 has been reported to enhance the occurrence and development of colon cancer (32). High RAD51 expression levels can induce the resistance of triple-negative breast cancer stem cells to chemotherapy drugs (33). In addition, it has been suggested that inhibition of RAD51 enhances the therapeutic effect of DDP on gastric cancer (34). Furthermore, CTBP1 influences the DDP resistance of breast cancer cells by activating RAD51 expression (19). The results of the present study demonstrated that CTBP1 expression was upregulated in DDP-resistant AGS/DDP and HGC-27/DDP gastric cancer cells. However, the DDP resistance of these cells was weakened following CTBP1 knockdown. Taken together, these results suggested that CTBP1 expression may influence the DDP resistance of gastric cancer cells. Notably, high levels of CTBP1 activated RAD51 expression in AGS/DDP cells, and overexpression of RAD51 abolished the CTBP1 knockdown-induced decrease in DDP resistance and apoptosis of DDP-resistant gastric cancer cells. These findings suggested that CTBP1 expression may be important for RAD51 foci formation and confer resistance to DDP to gastric cancer cells, which, in turn, may elevate the cancer cell DNA damage response. The effect of CTBP1 overexpression on the sensitivity to DDP in AGS and HCG-27 cells will be explored in future experiments and is a potential limitation to the present study.

The results of the present study demonstrated the effect of CTBP1 on the DDP resistance of gastric cancer cells. Taken together, the results suggested that CTBP1 strengthens the DDP resistance of gastric cancer cells by activating RAD51 expression, which may provide a novel therapy for the clinical treatment of patients with gastric cancer. However, the possibility that CTBP1 works in another way serving as a transcript corepressor of many types of protein and whether knockdown of CTBP1 changes other cell death pathways, such as necrosis and autophagy, will be investigated in future experiments and are limitations of the present study.

\section{Acknowledgements}

Not applicable.

\section{Funding}

Not applicable.

\section{Availability of data and materials}

The datasets used and/or analyzed during the current study are available from the corresponding author on reasonable request.

\section{Authors' contributions}

YLW and HYZ conceived the study, interpreted the data, designed the study, performed the experiments and analyzed the data. YLW wrote the manuscript and HYZ revised it. All authors read and approved the final manuscript. YLW and HYZ confirm the authenticity of all the raw data.

\section{Ethics approval and consent to participate}

Not applicable.

\section{Patient consent for publication}

Not applicable.

\section{Competing interests}

The authors declare that they have no competing interests. 


\section{References}

1. Van Cutsem E, Sagaert X, Topal B, Haustermans K and Prenen H: Gastric cancer. Lancet 388: 2654-2664, 2016.

2. Bray F, Ferlay J, Soerjomataram I, Siegel RL, Torre LA and Jemal A: Global cancer statistics 2018: GLOBOCAN estimates of incidence and mortality worldwide for 36 cancers in 185 countries. CA Cancer J Clin 68: 394-424, 2018.

3. Clevers H: The cancer stem cell: Premises, promises and challenges. Nat Med 17: 313-319, 2011.

4. Gupta GP and Massagué J: Cancer metastasis: Building a framework. Cell 127: 679-695, 2006.

5. Huang C, Chen R, Zheng F, Tang Y, Wang X, Chen Z and Lai X: Inhibitory role of ATF3 in gastric cancer progression through regulating cell EMT and stemness. Cancer Cell Int 21: 127, 2021.

6. Cats A, Jansen EPM, van Grieken NCT, Sikorska K, Lind P, Nordsmark M, Meershoek-Klein Kranenbarg E, Boot H, Trip AK, Swellengrebel HAM, et al: Chemotherapy versus chemoradiotherapy after surgery and preoperative chemotherapy for resectable gastric cancer (CRITICS): An international, open-label, randomised phase 3 trial. Lancet Oncol 19: 616-628, 2018.

7. Koizumi W, Tanabe S, Azuma M, Ishido K, Nishimura K, Sasaki T, Nakatani K, Higuchi K, Nakayama N and Katada C: Impacts of fluorouracil-metabolizing enzymes on the outcomes of patients treated with S-1 alone or S-1 plus cisplatin for first-line treatment of advanced gastric cancer. Int J Cancer 126: 162-170, 2010.

8. Liu Y, Bian T, Feng J, Qian L, Li X, Zhang Q, Zhang J, Jiang D, Liu J and Shi J: CtBP1 interacts with SOX2 to promote the growth, migration and invasion of lung adenocarcinoma. Oncol Rep 42: 67-78, 2019

9. Wang Y, Dai C, Zhou C, Li W, Qian Y, Wen J, Wang Y, Han B, Ma J, Xu J, et al: Benzotriazole enhances cell invasive potency in endometrial carcinoma through CTBP1-mediated epithelial-mesenchymal transition. Cell Physiol Biochem 44: 2357-2367, 2017.

10. Zhao C, Shen Y, Tao X, Xu J, Lu J, Liu C, Xu Z, Tang Q, Tao T and Zhang X: Silencing of CtBP1 suppresses the migration in human glioma cells. J Mol Histol 47: 297-304, 2016.

11. Moiola CP,De Luca P,ZalazarF, Cotignola J, Rodríguez-Seguí SA, Gardner K, Meiss R, Vallecorsa P, Pignataro O, Mazza O, et al: Prostate tumor growth is impaired by CtBP1 depletion in high-fat diet-fed mice. Clin Cancer Res 20: 4086-4095, 2014.

12. Porretti J, Dalton GN, Massillo C, Scalise GD, Farré PL, Elble R, Gerez EN, Accialini P, Cabanillas AM, Gardner K, et al: CLCA2 epigenetic regulation by CTBP1, HDACs, ZEB1, EP300 and miR-196b-5p impacts prostate cancer cell adhesion and EMT in metabolic syndrome disease. Int J Cancer 143: 897-906, 2018.

13. Dcona MM, Morris BL, Ellis KC and Grossman SR: CtBP-an emerging oncogene and novel small molecule drug target: Advances in the understanding of its oncogenic action and identification of therapeutic inhibitors. Cancer Biol Ther 18: 379-391, 2017.

14. Han Y, Bi Y, Bi H, Diao C, Zhang G, Cheng K and Yang Z: miR-137 suppresses the invasion and procedure of EMT of human breast cancer cell line MCF-7 through targeting CtBP1. Hum Cell 29: 30-36, 2016.

15. Zhou J, Su M, Zhang H, Wang J and Chen Y: miR-539-3P inhibits proliferation and invasion of gastric cancer cells by targeting CTBP1. Int J Clin Exp Pathol 12: 1618-1625, 2019.

16. Wang C, Wang M, Xing B, Chi Z, Wang H, Lie C and Dong H: $\mathrm{C}$-terminal of E1A binding protein 1 enhances the migration of gastric epithelial cells and has a clinicopathologic significance in human gastric carcinoma. Onco Targets Ther 12: 5189-5200, 2019.

17. Deng Y, Guo W, Xu N, Li F and Li J: CtBP1 transactivates RAD51 and confers cisplatin resistance to breast cancer cells. Mol Carcinog 59: 512-519, 2020.

18. Bhattacharyya A, Ear US, Koller BH, Weichselbaum RR and Bishop DK: The breast cancer susceptibility gene BRCA1 is required for subnuclear assembly of Rad51 and survival following treatment with the DNA cross-linking agent cisplatin. J Biol Chem 275: 23899-23903, 2000.
19. Feng Y, Wang D, Xiong L, Zhen G and Tan J: Predictive value of RAD51 on the survival and drug responsiveness of ovarian cancer. Cancer Cell Int 21: 249, 2021.

20. Mattiello L, Soliman Abdel Rehim S, Musella M, Sistigu A, Guarracino A, Vitale S, Corradi F, Galassi C, Sperati F, Manic G, et al: The targeting of MRE11 or RAD51 sensitizes colorectal cancer stem cells to CHK1 inhibition. Cancers (Basel) 13: 1957, 2021

21. Malka MM, Eberle J, Niedermayer K, Zlotos DP and Wiesmuller L: Dual PARP and RAD51 inhibitory drug conjugates show synergistic and selective effects on breast cancer cells. Biomolecules 11: 981, 2021.

22. Maranto C, Udhane V, Hoang DT, Gu L, Alexeev V, Malas K, Cardenas K, Brody JR, Rodeck U, Bergom C, et al: STAT5A/B blockade sensitizes prostate cancer to radiation through inhibition of RAD51 and DNA repair. Clin Cancer Res 24: 1917-1931, 2018.

23. Yang X, Zhang Q and Guan B: Circ_0110805 knockdown enhances cisplatin sensitivity and inhibits gastric cancer progression by miR-299-3p/ENDOPDI axis. OncoTargets Ther 13: 11445-11457, 2020.

24. Livak KJ and Schmittgen TD: Analysis of relative gene expression data using real-time quantitative PCR and the 2(-Delta Delta C(T)) method. Methods 25: 402-408, 2001.

25. Siegel RL, Miller KD and Jemal A: Cancer statistics, 2017. CA Cancer J Clin 67: 7-30, 2017.

26. Sun TT, He J, Liang Q, Ren LL, Yan TT, Yu TC, Tang JY, Bao YJ, Hu Y, Lin Y, et al: lncRNA GClncl promotes gastric carcinogenesis and may act as a modular scaffold of WDR5 and KAT2A complexes to specify the histone modification pattern. Cancer Discov 6: 784-801, 2016.

27. Sun Y, Ma J, Lin J, Sun D, Song P, Shi L, Li H, Wang R, Wang Z and Liu S: Circular RNA circ_ASAP2 regulates drug sensitivity and functional behaviors of cisplatin-resistant gastric cancer cells by the miR-330-3p/NT5E axis. Anti-Cancer Drugs: May 19, 2021 (Epub ahead of print). doi: 10.1097/CAD.0000000000001087.

28. Zhang S, Feng R, Yuan F, Luo Q, Chen X, Li N and Yang S: The therapeutic effects of dihydroartemisinin on cisplatin-resistant gastric cancer cells. Curr Pharm Biotechnol: Feb 16, 2021 (Epub ahead of print). doi: 10.2174/1389201022666210217114825.

29. Zhuo W, Liu Y, Li S, Guo D, Sun Q, Jin J, Rao X, Li M, Sun M, Jiang M, et al: Long noncoding RNA GMAN, Up-regulated in gastric cancer tissues, is associated with metastasis in patients and promotes translation of ephrin A1 by competitively binding GMAN-AS. Gastroenterology 156: 676-691.e11, 2019.

30. Chinnadurai G: The transcriptional corepressor CtBP: A foe of multiple tumor suppressors. Cancer Res 69: 731-734, 2009.

31. Chen X, Zhang Q, Dang X, Song T, Wang Y, Yu Z, Zhang S, Fan J, Cong F, Zhang W and Duan N: Targeting the CtBP1-FOXM1 transcriptional complex with small molecules to overcome MDR1-mediated chemoresistance in osteosarcoma cancer stem cells. J Cancer 12: 482-497, 2021.

32. He Y, He Z, Lin J, Chen C, Chen Y and Liu S: CtBP1/2 differentially regulate genomic stability and DNA repair pathway in high-grade serous ovarian cancer cell. Oncogenesis 10: 49, 2021.

33. Liu Y, Burness ML, Martin-Trevino R, Guy J, Bai S, Harouaka R, Brooks MD, Shang L, Fox A, Luther TK, et al: RAD51 mediates resistance of cancer stem cells to PARP inhibition in triple-negative breast cancer. Clin Cancer Res 23: 514-522, 2017.

34. He WL, Li YH, Hou WJ, Ke ZF, Chen XL, Lu LY, Cai SR, Song W, Zhang CH and He YL: RAD51 potentiates synergistic effects of chemotherapy with PCI-24781 and cis-diamminedichloroplatinum on gastric cancer. World J Gastroenterol 20: 10094-10107, 2014.

his work is licensed under a Creative Commons Attribution-NonCommercial-NoDerivatives 4.0 International (CC BY-NC-ND 4.0) License. 